$\mathbb{T}$ periodica polytechnica

\author{
Transportation Engineering \\ $37 / 1-2(2009) 29,32$ \\ doi: 10.3311/pp.tr.2009-1-2.05 \\ web: http://www.pp.bme.hu/tr \\ (c) Periodica Polytechnica 2009
}

RESEARCH ARTICLE

\section{Generalized polygonal Wankel engines}

Emil Molnár / Jenó Szirmai

Received 2008-12-02

\begin{abstract}
The trigonal Wankel engine is kinematically based on the motion where a circle $p_{m}$ of radius $3 d$, as the moving pole curve, rolles on the circle $p_{s}$ of radius $2 d$, as the standing pole curve in the interior. Then the regular trigonal rotor with circumcircle of radius $\rho>3 d$, fixed concentrically to the moving pole circle, describes its orbit curve $c_{\rho}$. This orbit curve $c_{\rho}$ is crutial in forming the engine space.

Answering a question of István Revutzky, we prove (and animate by computer) that $c_{\rho}$ is a convex curve iff $\rho$ bigger or equal to $9 \mathrm{~d}$. The parallel curve $c_{\rho+r}$ with distance $r$ will be the solution to the engine space if the triangle rotor touches $c_{\rho+r}$ with small roller circles of radius $r$ centred in the vertices of the triangle.
\end{abstract}

All these concepts will be generalized - with animation - to a $k$-gonal rotor $(2<k$ natural number $)$ in a unified way.

\section{Keywords}

Wankel engine $\cdot$ kinematical geometry $\cdot$ trochoidal motion

\section{Acknowledgement}

The authors thank Colleagues István Emöd and Liviu Finichiu for the fruitful conversations and Professor Hellmuth Stachel for kind hints to kinematical references.

\section{Emil Molná}

Institute of Mathematics, Department of Geometry BME, H-1521 Budapest, Hungary

e-mail: emolnar@math.bme.hu

\section{Jenő Szirmai}

Institute of Mathematics, Department of Geometry BME, H-1521 Budapest, Hungary

e-mail: szirmai@math.bme.hu

\section{Introduction}

We continue the abstract with a few of historical remarks, for these we thank engineer professor colleagues István Emőd and Liviu Finichiu, furthermore Professor Hellmuth Stachel (Vienna Unyversity of Technology).

After studying the literature of the topic (e.g. [1,8] and cited references there) it turned out that Wankel himself thought of this generalization and of other peculiar constructions. Although Wankel engine is not effective enough, its mechanism is attractive still nowadays.

About 1970 the envelopes of trochoids under trochoidal motion were intensively studied by Austrian and German geometricians. Here we cite only [3], [6], [9] and the references there. The phenomenon of convex engine space seems to be new in this paper, and may be the simple short derivation of different possibilities by mathematical tools cause some enjoyments for the interested reader.

Optimization of such type of Wankel engines seems to be an open problem yet!

\section{Formulation of the problem, results}

Let a regular $k$-gon, with centre $Q$ and circumcircle of radius $\rho$, glide on an orbit curve $c_{\rho}$ of centre $O$ with similarity parameter $O Q=d$ constant. Then any vertex $P_{i}$ moves by time $t$ as follows:

$$
\overrightarrow{O P_{i}(t)}=\overrightarrow{O Q(t)}+\overrightarrow{Q(t) P_{i}(t)}=d \mathbf{e}(k t)+\rho \mathbf{e}(t) .
$$

Here we assume unit angular velocity for unit vector $\mathbf{e}(t)$ of angle $t$ (with $\mathbf{i}=\mathbf{e}(0)) . \overrightarrow{O Q(t)}=d \mathbf{e}(k t)$ is then a good choice for the requirements that $P_{i}$ describes the same curve $c_{\rho}$ for $i=1,2, \ldots, k$. Indeed, the substitutions

$$
t^{\prime} \mapsto t+\frac{2 \pi i}{k}, \quad i=1,2, \ldots, k
$$

lead to the same geometric description in Eq. (1). In the or-

\footnotetext{
${ }^{1}$ Motivated and inspired by ideas and instruments of István Revuczky (Dunaújváros).

Lectured at the $5^{\text {th }}$ Conference "Konstruktive Geometrie" Balatonföldvár (Hungary) "Hotel Jogar" 05-09. 09. 2005.

Supported by DAAD 2008: Multimedia Technology for Mathematics and Computer Science Education.
} 


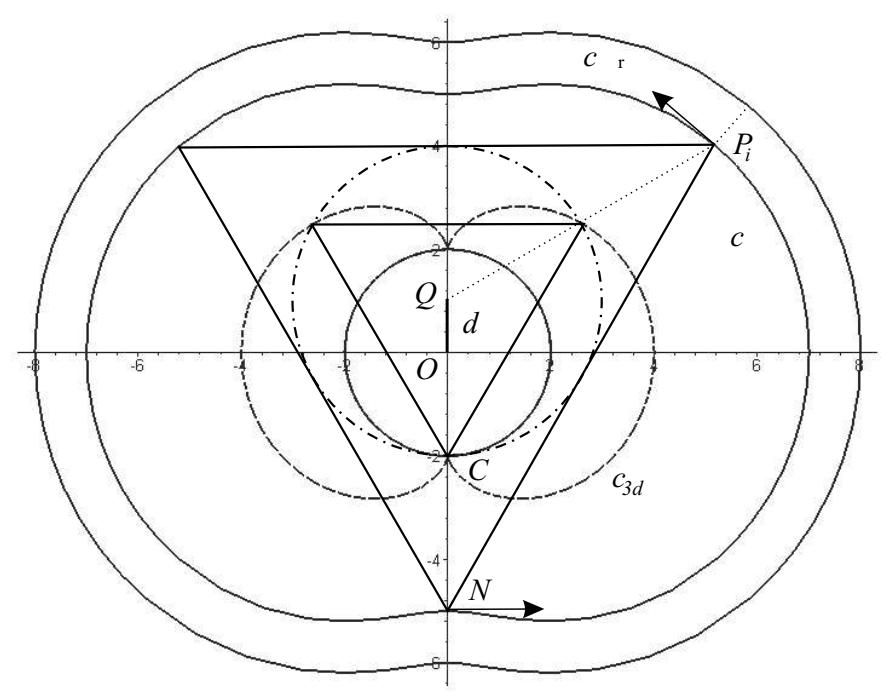

Fig. 1. The principles of 3-gonal Wankel engine.

thonormal coordinate system $(O ; \mathbf{i}, \mathbf{j})$ the Eq. (1) will have the form for the vectors $\mathbf{c}_{\rho}(t)$ pointing to the points of the so-called trochoid curve $c_{\rho}$ :

$$
\begin{aligned}
& \mathbf{c}_{\rho}(t): x=d \cos k t+\rho \cos t, \\
& y=d \sin k t+\rho \sin t, \quad 0 \leq t \leq 2 \pi ; \\
& \text { and } \dot{\mathbf{c}}_{\rho}(t): \dot{x}=-k d \sin k t-\rho \sin t, \\
& \dot{y}=k d \cos k t+\rho \cos t ; \\
& \ddot{\mathbf{c}}_{\rho}(t): \ddot{x}=-k^{2} d \cos k t-\rho \cos t, \\
& \ddot{y}=-k^{2} d \sin k t-\rho \sin t
\end{aligned}
$$

express the velocity and acceleration vectors of $\mathbf{c}_{\rho}(t)$, respectively. Thus the absolute value of the velocity is

$$
\begin{aligned}
& \left|\dot{\mathbf{c}}_{\rho}(t)\right|=\sqrt{k^{2} d^{2}+\rho^{2}+2 k \rho d \cos (k-1) t}>0, \\
& \text { for any } t \text {, if } \rho>k d .
\end{aligned}
$$

Therefore the parallel curve $c_{\rho+r}$ can be given by coordinates

$$
X=\frac{1}{\left|\dot{\mathbf{c}}_{\rho}(t)\right|}\left\{d\left[\left|\dot{\mathbf{c}}_{\rho}(t)\right|+r k\right] \cos k t+\rho\left[\left|\dot{\mathbf{c}}_{\rho}(t)\right|+r\right] \cos t\right\},
$$

$$
Y=\frac{1}{\left|\dot{\mathbf{c}}_{\rho}(t)\right|}\left\{d\left[\left|\dot{\mathbf{c}}_{\rho}(t)\right|+r k\right] \sin k t+\rho\left[\left|\dot{\mathbf{c}}_{\rho}(t)\right|+r\right] \sin t\right\} .
$$

This $c_{\rho+r}$ and $c_{\rho}$ as well will be of non-negative signed curvature, and both will be convex by Eq. (3), iff

$$
\rho \geq k^{2} d
$$

Thus, for $k=3, d=1, \rho=9$, e.g. $r=1$, we shall have an ellipsis like motor, called elliptor by István Revuczky (Fig.2),

$$
a:=\rho+d+r=11, \quad b:=\rho-d+r=9
$$

will be the half axes of the elliptor that contains the corresponding ellipsis.

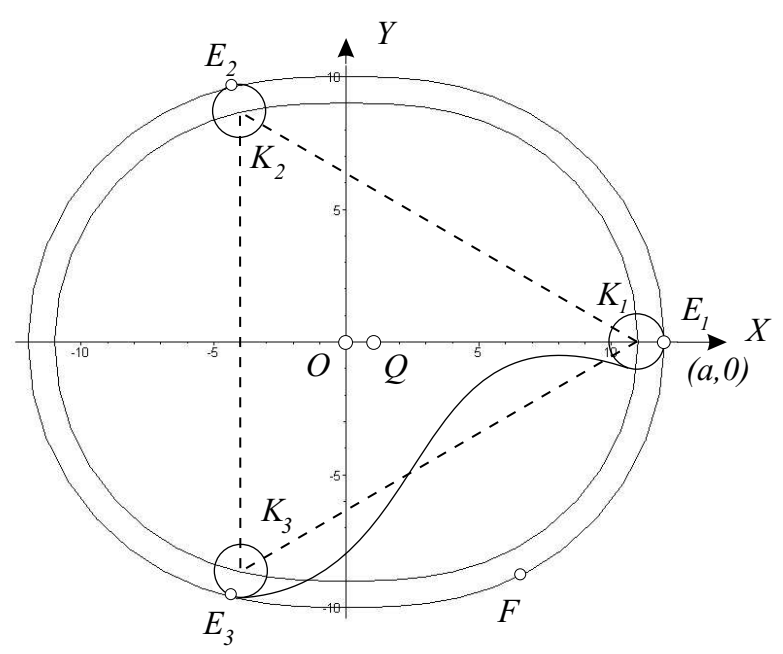

Fig. 2. The convex "elliptor" engine space with a hypothetic rotor profile and fire point $F$.

This last fact does not fit Revuczky's imagination, but convexity may have advantages for constructing a new type of engine. The profile of rotor can also be varied so that the "explosion" from a fire point near $F$, e.g. in Fig. 2, be optimal by its effect.

The equation of the engine profile in Eq. (??) is very general by its parameters $k, d, \rho, r$. The exact computations for the time variable $t$ are hopeless without computer. Animation of the movements is still timely problem, [4] is a first step, only.

\section{The pole curves}

To describe the motion of any point $X$ of the moving regular $k$-gonal rotor (in Fig. 3, $k=3$ ), we introduce the fixed coordinate system $\left(O ; \mathbf{e}_{1}, \mathbf{e}_{2}\right)$ and the moving coordinate system $\left(Q ; \mathbf{a}_{1}, \mathbf{a}_{2}\right)$ in the sense of Section 1 :

$$
\begin{aligned}
& \overrightarrow{O Q}=\left(\begin{array}{ll}
\mathbf{e}_{1} & \mathbf{e}_{2}
\end{array}\right)\left(\begin{array}{l}
d \cos k t \\
d \sin k t
\end{array}\right), \\
& \left(\begin{array}{ll}
\mathbf{a}_{1} & \mathbf{a}_{2}
\end{array}\right)=\left(\begin{array}{ll}
\mathbf{e}_{1} & \mathbf{e}_{2}
\end{array}\right)\left(\begin{array}{cc}
\cos t & -\sin t \\
\sin t & \cos t
\end{array}\right), \\
& \overrightarrow{Q X}=\mathbf{x}=\left(\begin{array}{ll}
\mathbf{a}_{1} & \mathbf{a}_{2}
\end{array}\right)\left(\begin{array}{l}
x^{1} \\
x^{2}
\end{array}\right), \\
& \overrightarrow{O X}=\mathbf{y}=\left(\begin{array}{ll}
\mathbf{e}_{1} & \mathbf{e}_{2}
\end{array}\right)\left(\begin{array}{l}
y^{1} \\
y^{2}
\end{array}\right)= \\
& =\overrightarrow{O Q}+\overrightarrow{Q X}=\left(\begin{array}{ll}
\mathbf{e}_{1} & \mathbf{e}_{2}
\end{array}\right)\left(\begin{array}{l}
d \cos k t \\
d \sin k t
\end{array}\right)+ \\
& \left(\begin{array}{ll}
\mathbf{e}_{1} & \mathbf{e}_{2}
\end{array}\right)\left(\begin{array}{cc}
\cos t & -\sin t \\
\sin t & \cos t
\end{array}\right)\left(\begin{array}{l}
x^{1} \\
x^{2}
\end{array}\right) \\
& \left(\begin{array}{l}
y^{1}(t) \\
y^{2}(t)
\end{array}\right)=\left(\begin{array}{l}
d \cos k t \\
d \sin k t
\end{array}\right)+\left(\begin{array}{cc}
\cos t & -\sin t \\
\sin t & \cos t
\end{array}\right)\left(\begin{array}{l}
x^{1} \\
x^{2}
\end{array}\right) .
\end{aligned}
$$

Here $\left(x^{1}, x^{2}\right)^{T}$ is fixed, but $\left(y^{1}(t), y^{2}(t)\right)^{T}$ depends on the time, because $\overrightarrow{O Q}$ and $\left(\mathbf{a}_{1}, \mathbf{a}_{2}\right)$ move. We use row-column multiplication, lower-upper index convention and transpose (by upper $T$ ) for saving place. 


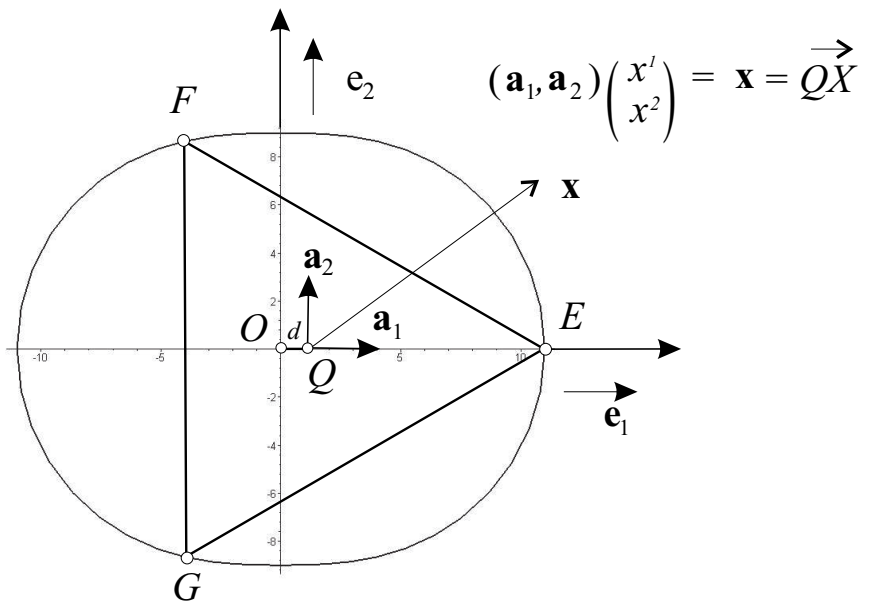

Fig. 3. Derivation of pole curves and orbits.

For an instantaneous pole $X\left(x^{1}, x^{2}\right)^{T}$, its velocity in the fixed frame is zero, $\dot{\mathbf{y}}:=\frac{\mathrm{d}}{\mathrm{d} t} \overrightarrow{O X}=\mathbf{0}$ yields from the last Eq. 99p:

$\left(\begin{array}{l}0 \\ 0\end{array}\right)=\left(\begin{array}{l}\dot{y}^{1}(t) \\ \dot{y}^{2}(t)\end{array}\right)=\left(\begin{array}{c}-k d \sin k t \\ k d \cos k t\end{array}\right)+\left(\begin{array}{cc}-\sin t & -\cos t \\ \cos t & -\sin t\end{array}\right)\left(\begin{array}{l}x^{1} \\ x^{2}\end{array}\right)$.

In a fixed moment $t=\tau$ this serves us the pole $\left(x^{1}(\tau), x^{2}(\tau)\right)^{T}$ as a solution of Eq.99(). Finally we get

$$
\left(\begin{array}{l}
x^{1}(\tau) \\
x^{2}(\tau)
\end{array}\right)=-k d\left(\begin{array}{c}
\cos (k-1) \tau \\
\sin (k-1) \tau
\end{array}\right) \text { the moving pole curve } p_{m}
$$

in the moving frame $\left(Q, \mathbf{a}_{1}, \mathbf{a}_{2}\right)$. Formula $(10)$ describes $p_{m}$ as a moving circle of radius $k d$ with centre $Q$. Substituting (10) into the last equation of $(9)$, we obtain

$$
\left(\begin{array}{l}
y^{1}(\tau) \\
y^{2}(\tau)
\end{array}\right)=-(k-1) d\left(\begin{array}{c}
\cos k \tau \\
\sin k \tau
\end{array}\right) \text { the fixed pole curve } p_{s}
$$

in the standing frame $\left(O, \mathbf{e}_{1}, \mathbf{e}_{2}\right)$. This is a circle of radius $(k-1) d$ with centre $O$.

Indeed, the moving pole curve $p_{m}$ rolles on the standing pole curve $p_{s}$. Meanwhile, the rotor, fixed to the moving pole curve $p_{m}$, can be studied (Fig. 1], animated (Home pages [?4]-[?5]), etc.

Thus the profile of the engine space, the rotor and fire point $F$ can be formed optimally by experiments or by other theoretical tools (?), as we sketched it for $k=3$, e.g. in Fig. 2.

\section{Concluding remarks for k-gonal rotors, $k>3$}

Our Fig. 4 (see also Fig. 5) indicates the situation for $k=$ $4, d=1=r, \rho=k d=4$. The moving pole curve $p_{m}$ is a circle of the critical radius $k d=4$, with centre $Q$. The fixed pole curve $p_{s}$ is of radius $(k-1) d=3$. The regular $k(=4)$-gonal rotor is fixed to the moving pole curve $p_{m}$. The $k-1(=3)$ fold rotatory symmetry of any orbit is obvious. For $\rho \geq k^{2} d$ (=16) we shall have convex orbit $c_{\rho}$, so convex motor space (Fig. 5). The parallel curve $c_{\rho+r}$ of distance $r$ will be formed for a $k(=4)$-gonal rotor with rolling circles of radius $r(=1)$ in the vertices. Our animation [4] shows the motion.

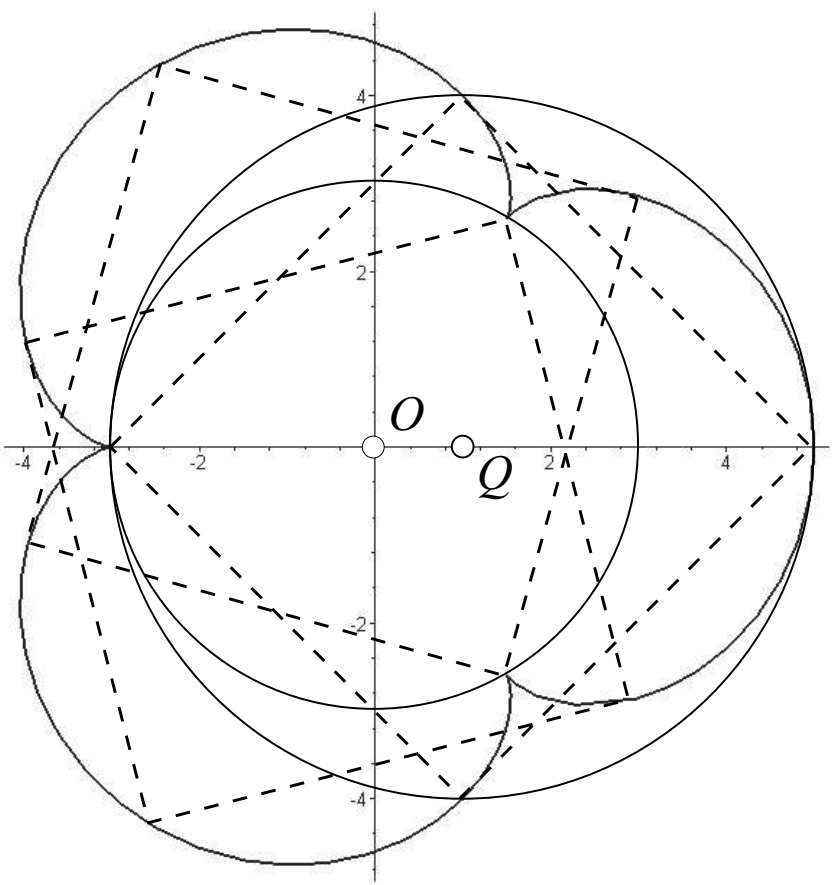

Fig. 4. The principles for $k(=4)$-gonal rotor.

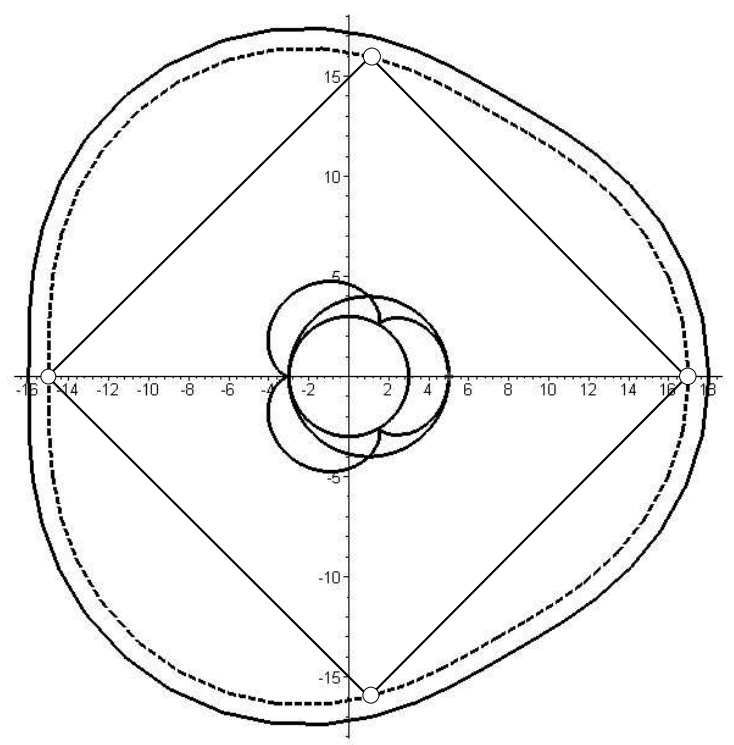

Fig. 5. The convex engine space for $k(=4)$-gonal rotor.

$k=3$ is logically the simplest solution for the engine with $k=3$ areas of periodic functions, as "explosion", expansion emission and suction compression of fuel. One can imagine that $k$ (> 3)-times explosions in a period and more functions with fuel can also be advantageous for an engine.

It is time to experiment with it! Nowadays, this experiment may happen by computer animations as well. We know Mazda's work with Wankel engines. May be for small engines, 4-gonal convex engine has chance in the future (Figs. 4, 55. 


\section{References}

1 Bensinger W D, Rotationskolben - Verbrennungsmotoren, Springer - Verlag, Berlin-Heidelberg-New York, 1973.

2 Bottema O, Roth B, Theoretical Kinematics, North Holland Publishing Company, Amsterdam, 1979.

3 Meyer P, Über Hüllkurven von Radlinien, Arch. der Math. 18 (1967), 651662.

4 Molnár E, Szirmai J, Generalized polygonal Wankel engines, animation, available at http://www.math.bme.hu/ geom

5 available at http://www. der-wankelmotor.de

6 Stachel H, Hüllbahnen von Trochoiden bei Trochoidenbewegungen, Math. Nachrichten 46 (1970), 189-208.

7 Strommer Gy, Geometria, Múszaki Könyvkiadó, Budapest, 1985.

8 Wankel F, Die Anzahl der Zylinder und Kammern bei durch satzgleichen Viertaktmotoren mit Hubkolben und mit Rotationskolben der Trochoiden bauart, MTZ Motortechnische Zeitschrift 25 (1964), no. 12.

9 Wunderlich W, Ebene Kinematik, Bibliographisches Institut, MannheimWien-Zürich, 1970. 